\title{
Intense beam of metastable Muonium
}

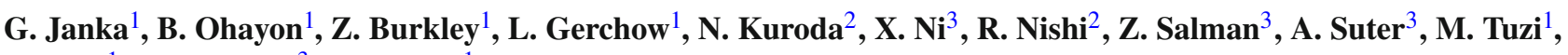 \\ C. Vigo ${ }^{1}$, T. Prokscha ${ }^{3}$, P. Crivelli ${ }^{1, \mathrm{a}}$ (D) \\ ${ }^{1}$ Institute for Particle Physics and Astrophysics, ETH Zürich, 8093 Zurich, Switzerland \\ ${ }^{2}$ Institute of Physics, The University of Tokyo, Tokyo 153-8902, Japan \\ ${ }^{3}$ Laboratory for Muon Spin Spectroscopy, Paul Scherrer Institute (PSI), 5232 Villigen, Switzerland
}

Received: 19 May 2020 / Accepted: 24 August 2020 / Published online: 1 September 2020

(C) The Author(s) 2020

\begin{abstract}
Precision spectroscopy of the Muonium Lamb shift and fine structure requires a robust source of $2 \mathrm{~S}$ Muonium. To date, the beam-foil technique is the only demonstrated method for creating such a beam in vacuum. Previous experiments using this technique were statistics limited, and new measurements would benefit tremendously from the efficient $2 \mathrm{~S}$ production at a low energy muon $(<20 \mathrm{keV})$ facility. Such a source of abundant low energy $\mu^{+}$has only become available in recent years, e.g. at the Low-Energy Muon beamline at the Paul Scherrer Institute. Using this source, we report on the successful creation of an intense, directed beam of metastable Muonium. We find that even though the theoretical Muonium fraction is maximal in the low energy range of $2-5 \mathrm{keV}$, scattering by the foil and transport characteristics of the beamline favor slightly higher $\mu^{+}$energies of $7-10 \mathrm{keV}$. We estimate that an event detection rate of a few events per second for a future Lamb shift measurement is feasible, enabling an increase in precision by two orders of magnitude over previous determinations.
\end{abstract}

\section{Introduction}

Muonium (M) is the bound state of a positive muon $\left(\mu^{+}\right)$ and an electron, two particles devoid of internal structure. Therefore, and in contrast to hydrogen, theory and experiment with $\mathrm{M}$ can be compared free of finite-size and nuclear effects [1]. Testing bound state quantum electrodynamics (QED) in the muonic sector is highly motivated by the inconsistencies which have arisen there, e.g. the deviation of the measured anomalous magnetic moment of the muon from its theoretical value [2], and the difference between the proton radius as measured by laser spectroscopy of muonic hydrogen [3] and several experiments in electronic hydrogen [4-6]. However, the puzzle is arguably nearing its solution [7-9].

a e-mail: crivelli@phys.ethz.ch (corresponding author)
To this day, precision experiments with $\mathrm{M}$ only utilized its ground-state $[10,11]$. The $1 \mathrm{~S}-2 \mathrm{~S}$ transition was measured by pulsed laser spectroscopy $[12,13]$, putting tight bounds on the muon-electron charge ratio. A future precision measurement using a CW laser is planned by the Mu-MASS experiment [14]. The measurement of the ground-state hyperfine structure currently determines the muon magnetic moment with the highest precision [15], with an improvement underway by the MUSEUM collaboration [16,17]. However, the methods used for $\mathrm{M}$ production in these measurements do not produce sufficient $\mathrm{M}(2 \mathrm{~S})$ in vacuum, and so cannot be used to study transitions from long-lived excited states. These include the $n=2$ Lamb shift [18,19] and fine-structure [20], which were measured previously in M. Other transitions probed in hydrogen with fast beams may be considered as well [21-24]. Similar to hydrogen, metastable $\mathrm{M}$ can be formed with the beamfoil technique [25], and indeed $\mathrm{M}(2 \mathrm{~S})$ was first observed at the TRIUMF cyclotron accelerator using sub-surface $\mu^{+}$at $2.1 \mathrm{MeV}$, impinging on gold and aluminum foils [26], followed by an observation at the Los Alamos Meson Physics Facility (LAMPF) [27]. In the Born approximation, production of $\mathrm{M}$ with this beam-foil technique is expected to be comparable to hydrogen with protons at the same velocity [28], favouring energies of several keV [29,30]. For this reason, the TRIUMF and LAMPF muon beams had to be significantly lowered in energy by degrader foils with the price of losing roughly half of the beam intensity. This resulted in a wide angular distribution for the emitted M [31]. The broad energy distribution of the degraded muon beam, extending from keVs to MeVs, resulted in a wide $\mathrm{M}$ energy distribution peaking at low energy and extending up to $20 \mathrm{keV}$, which severely limited the overall $\mathrm{M}$ yield.

At TRIUMF, the estimated production rate of $\mathrm{M}(2 \mathrm{~S})$ per incident muon was $0.08 \%$ [18]. This low efficiency, combined with a large divergence of the beam, and a large muonrelated background, resulted in a maximal detection rate of 
Fig. 1 The experimental setup installed at the end of the LEM beamline (see text), lengths at the bottom are not to scale. To the right there is an example of the time distribution of particles reaching the Stop-MCP in coincidence with the Tag-MCP. The paths of $\mathrm{M}$ and $\mu^{+}$are not parallel for visualization reasons

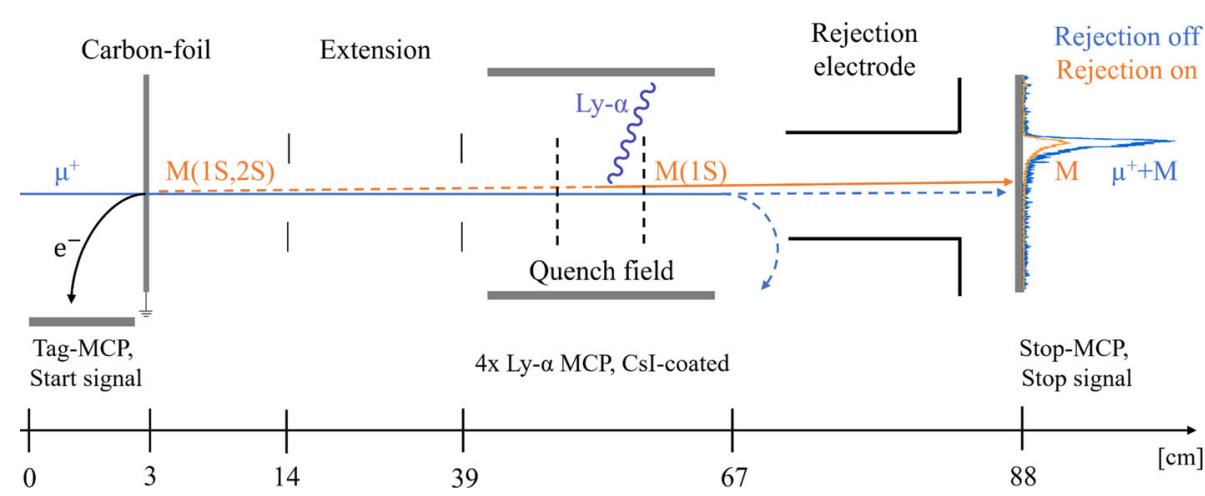

a few events per hour [32]. This limited the precision of the Lamb shift measurement to $1 \%$ [18]. Another experiment was conducted in parallel at the LAMPF accelerator, using similar methods and arriving at a comparable uncertainty of $2 \%$ [19].

To enable the next generation of precision measurements with metastable $\mathrm{M}$, we set out to solve the main limitation affecting previous campaigns. In this communication, we report on the efficient creation and detection of a nearly collinear $\mathrm{M}(2 \mathrm{~S})$ beam by the beam-foil technique. Employing the slow $\mu^{+}$from the Low-Energy-Muon (LEM) beamline at the Paul Scherrer Institute (PSI) [33,34] with energies $<10 \mathrm{keV}$ allowed us to directly use a thin $(\sim 15 \mathrm{~nm})$ carbon foil as a conversion target without the necessity of any prior beam degradation. By tagging each muon and measuring its time-of-flight (TOF), we report for the first time the $\mathrm{M}$ creation efficiency over well-defined exit energies ranging from 2 to $8 \mathrm{keV}$. Through quenching the metastable $2 \mathrm{~S}$ state to the short-lived $2 \mathrm{P}$ state in a static electric field and detecting the emitted Lyman- $\alpha$ photons, we extract the $2 \mathrm{~S}$ fraction and compare with estimation from the literature. Combining our results with particle tracing simulations, we were able to quantify the $\mathrm{M}(2 \mathrm{~S})$ beam parameters. These parameters enable a realistic estimation of the achievable event rate for a future Lamb shift measurement. We conclude that a significant improvement over the state-of-the-art is within reach.

\section{Experimental setup}

The LEM beamline at PSI generates low energy muons by moderating a surface $\mu^{+}$beam (4 MeV energy) from the $\mu \mathrm{E} 4$ beamline with a silver foil coated with a thick layer of a solid noble gas mixture [34-36]. For the experiment conducted here, a solid neon moderator was used, allowing the formation of a slow, monoenergetic $(2-12 \mathrm{keV}) \mu^{+}$beam. In this energy regime, hydrogen formation data $[37,38]$ suggests a high production rate of $\mathrm{M}$, some in metastable states, by impinging $\mu^{+}$on a foil.
In the measurements performed, three different incident energies $E_{\text {inc }}$ of $5,7.5$, and $10 \mathrm{keV}$ were chosen. For each $E_{\text {inc }}$, the beamline parameters were optimized, utilizing the Geant4-based musrSim simulation $[39,40]$. Therefore, while the highest $\mu^{+}$to $\mathrm{M}$ conversion efficiencies are expected at the lowest $\mu^{+}$energies, we gain in transportation and detection efficiency with increasing energy. An energy of $5 \mathrm{keV}$ appeared to be a lower threshold in this regard.

The experimental setup for generating and characterizing the $\mathrm{M}$ and $\mathrm{M}(2 \mathrm{~S})$ beam is shown in Fig. 1. Muons from the LEM beamline are directed onto a carbon foil. A fraction of the $\mu^{+}$captures an electron while passing through the foil, forming $\mathrm{M}$ primarily in the ground and $n=2$ states. The carbon foil can also be used to tag incoming $\mu^{+}$. When an incoming muon hits the foil, on average one secondary electron (SE) is released upstream [41]. This SE is guided to a microchannel plate (Tag-MCP), giving the start time for the experiment. Upon transmission, SE formed downstream might also be created. To prevent these electrons from creating false signals, the front plate of each subsequent MCP detector is biased with a negative high voltage in order to reject them.

The beam emerging from the foil, which is grounded to prevent quenching of any $\mathrm{M}$ formed in the $2 \mathrm{~S}$ state, propagates in a field-free region, and then through an electrical quenching region formed by two ring electrodes that mixes the $2 \mathrm{~S}$ and $2 \mathrm{P}$ states. The field at the center is 400 $\mathrm{V} / \mathrm{cm}$. Unlike the metastable $2 \mathrm{~S}$ state, the $2 \mathrm{P}$ state is shortlived ( $\left.\tau_{2 P} \approx 1.6 \mathrm{~ns}\right)$ and relaxes to the ground state within a few nanoseconds, emitting a photon of $122 \mathrm{~nm}(\mathrm{Ly}-\alpha)$. This photon can be detected by four CsI-coated MCPs (Ly$\alpha$-MCP) surrounding the quenching area. The beam exiting the quenching region, now containing predominantly $\mathrm{M}(1 \mathrm{~S})$ and $\mu^{+}$, reaches a rejection electrode at high voltage $\left(E_{\text {inc }}\right.$ $+1 \mathrm{kV})$ that only allows passage of $\mathrm{M}(1 \mathrm{~S})$ by blocking $\mu^{+}$. The surviving $\mathrm{M}(1 \mathrm{~S})$ impinge onto an MCP (Stop-MCP), providing the stop signal. 


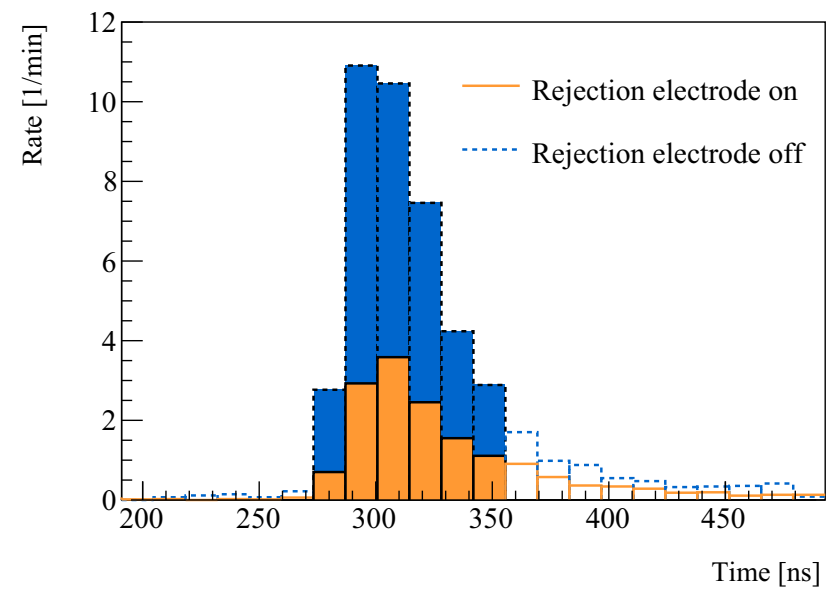

Fig. 2 Histograms obtained from the dataset of $10 \mathrm{keV} E_{\text {inc }}$ after background subtraction. The solid line data is with rejection field on and corresponds to pure $\mathrm{M}$ signal. The dotted data is with rejection electrode off and corresponds to $\mathrm{M}$ and $\mu^{+}$signal. The filled bins were used to extract $\mathrm{M}$ fractions, whereas the hollow bins were ignored due to large statistical uncertainty and additional background

\section{Muonium fractions at different energies}

The fraction of $\mathrm{M}$ formed out of the incident muon beam, $f_{\mathrm{M} / \mu^{+}}$, is extracted from coincidence events between the Tag- and Stop-MCP with the rejection electrode turned on or off for different $E_{\text {inc. The TOF spectra for rejection off }}$ $\left(\mathrm{M}+\mu^{+}\right)$and rejection on $(\mathrm{M})$, after a subtraction of a constant background of 0.1 counts $/ \mathrm{min}$, are divided into time bins, with the results for $10 \mathrm{keV}$ incident $\mu^{+}$shown in Fig. 2.

An extension stage can be added between the carbon foil and the quenching region to extend the travelling distance. The resulting increase in TOF allows the extraction of the velocity and thus energy distributions of both $\mu^{+}$and $\mathrm{M}$ after the foil, which are not known a priori. Additionally, the extension stage ensures that all $2 \mathrm{P}$ states, as well as higher lying states produced in the foil [42], decay prior to reaching the quenching region. For $10 \mathrm{keV}$ incident $\mu^{+}$, the spectra were measured with and without the extension stage (Fig. 3).

A Landau distribution was found to describe well the TOF spectra. In addition to the length of the stage and the entire distance between foil and Stop-MCP, the time offset of the detection system was determined with a linear fit to be $t_{0}=$ $51 \pm 4 \mathrm{~ns}$. As the extension stage was always present during the measurements with $E_{\text {inc }}$ of 5.0 and $7.5 \mathrm{keV}, t_{0}$ and the total length were used to convert these TOF spectra to the energy distributions presented in Fig. 4.

We found that the most probable energy loss in the foil is 2.3-3.0 keV (see Table 1). The foil thickness can be derived from the results for the Most Probable Energy (MPE) and the corresponding energy distributions by comparing them with the LEM Geant 4 simulation, in which an effective, calibrated interaction with the foil is implemented [40]. We find that a

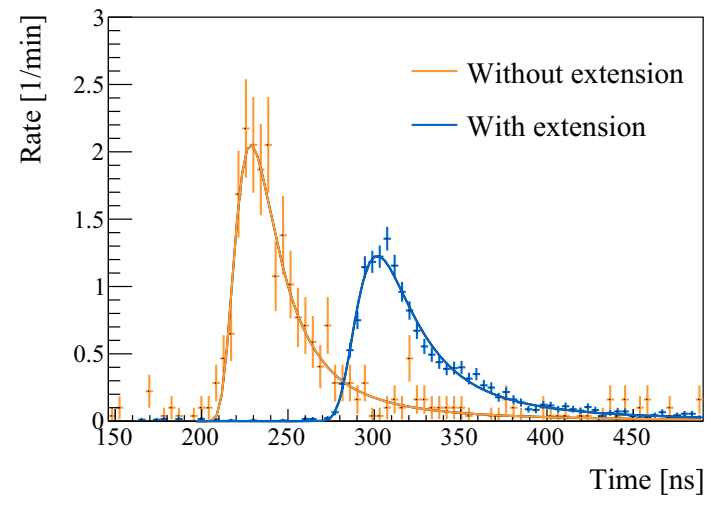

Fig. 3 TOF distributions of $\mathrm{M}$ for $10 \mathrm{keV} \mu^{+}$incident on the foil, with (dark blue) and without (light orange) extension stage. The errors on the rate are from statistics. A Landau distribution was used for fitting the spectra

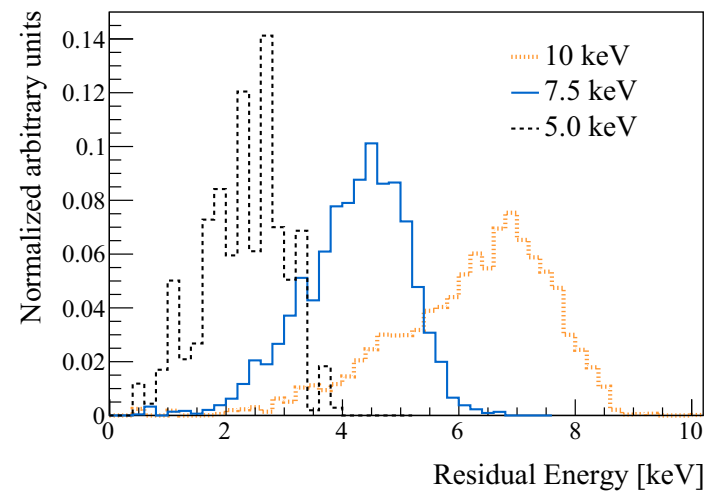

Fig. 4 Energy distributions of M reaching the Stop-MCP, measured at three different $E_{\text {inc }}$. Areas are normalized to 1

thickness of $15 \mathrm{~nm}$ is most probable, which is more than the $10 \mathrm{~nm}$ specified by the manufacturer. This fact is not surprising considering the differences between the nominal and derived carbon foil thicknesses determined in [43].

From our knowledge of the $\mathrm{M}$ fractions and residual energy distributions, we can determine the $\mathrm{M}$ conversion rate of our foil in this low incident muon energy range. The results are shown in Fig. 5. The errors in the fractions are dominated by statistics, and those in the mean residual energy are correlated and arise from the uncertainty of $t_{0}$. Our results demonstrate that in the energy range probed, a high conversion rate to $\mathrm{M}$ is achieved, leading to the expectation that a sizeable amount of $\mathrm{M}(2 \mathrm{~S})$ is also produced [37].

\section{Determination of $M(2 S)$ fraction}

The fraction of $\mathrm{M}(2 \mathrm{~S})$ of the total $\mathrm{M}$ produced, $f_{2 \mathrm{~S} / \mathrm{M}}$, is extracted from triple coincidence events between the Tag, Ly$\alpha$, and Stop-MCPs with the quenching electrodes turned on or off, while keeping the rejection electrode turned on. The rate 


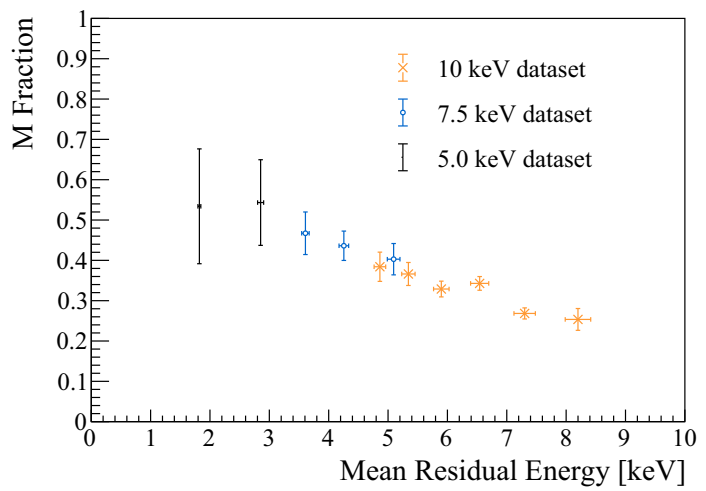

Fig. $5 \mathrm{M}$ fraction measured as a function of residual energy after the foil

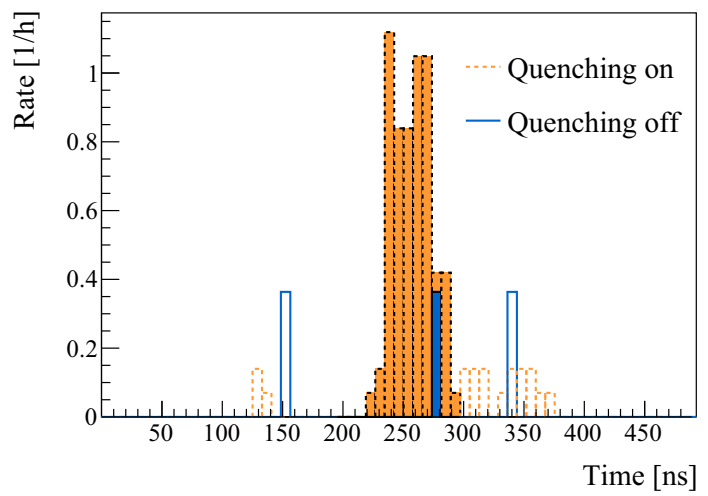

Fig. 6 Time-of-flight distributions of the counts in the Ly- $\alpha$-MCPs, obtained from the triple coincidence dataset of $10 \mathrm{keV} E_{\text {inc }}$. The dotted data is with quenching electrodes turned on, the solid data is with quenching off. The coloured area is the time window of interest, where the Ly- $\alpha$ signal is to be expected

of triple coincidence events, $R_{\mathrm{T}}$, indicative of $\mathrm{M}(2 \mathrm{~S})$, is then compared to the rate of double coincidence events between the Tag and Stop-MCPs, $R_{\mathrm{D}}$, indicative of M. The clear triplecoincidence Ly- $\alpha$ signal is shown in Fig. 6 for $E_{\text {inc }}$ of $10 \mathrm{keV}$. The Ly- $\alpha$ signal can be seen in the expected time window calculated using the energy distributions from Fig. 4 and the distance, including the extension stage, between foil and the quenching area. Taking into account the photon detection efficiencies, the resulting fraction of $\mathrm{M}(2 \mathrm{~S})$ out of the total $\mathrm{M}$ is

$f_{2 \mathrm{~S} / \mathrm{M}}=\frac{R_{\mathrm{T}}}{R_{\mathrm{D}} \cdot \varepsilon_{\mathrm{QG}} \cdot \varepsilon_{\mathrm{MCP}}}$,

where $\varepsilon_{\mathrm{MCP}}$ stands for the Ly- $\alpha$ detection efficiency of the $\mathrm{MCP}$, and $\varepsilon_{\mathrm{QG}}$ for the combined efficiency for quenching as well as the solid angle covered by the detectors. The quenching and geometrical efficiency of the Ly- $\alpha$ detection stage are correlated, and depend on the M velocity, since the position the $\mathrm{M}(2 \mathrm{~S})$ reaches before quenching affects the solid

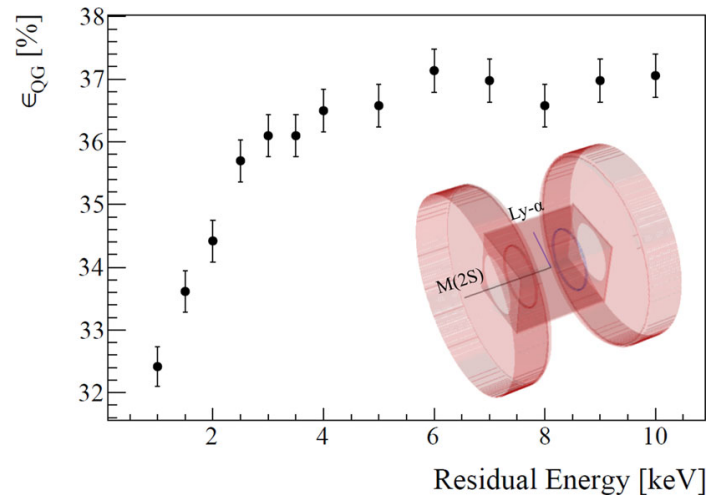

Fig. 7 Results of Monte-Carlo simulation for the quenching and geometrical efficiency as a function of energy. The inset portrays a simulated valid event where $\mathrm{M}(2 \mathrm{~S})$ enters the detection region, is quenched by the static field created by the two circular electrodes, and emits a photon which reached one of the detectors

angle. To determine $\varepsilon_{\mathrm{QG}}$, we performed a full 3D MonteCarlo simulation of the particle motion and photon emission inside the static electric field using the SIMION 8.1 package [44]. The position distribution of the particles at the detector entrance was taken from the GEANT4 beamline simulation with the calibrated foil thickness, taking into account the coincidence detection in the Stop-MCP. Additionally, the anisotropy of the photon emission relative to the electric field direction [45], and the transparency of the grids on the detectors, were included. The total efficiency is shown in Fig. 7. Folding it with the measured energy distributions, we get $\varepsilon_{\mathrm{QG}}=36.4 \pm 0.3 \%$ and $\varepsilon_{\mathrm{QG}}=37.0 \pm 0.3 \%$, for $E_{\mathrm{inc}}$ of 7.5 and $10 \mathrm{keV}$, respectively.

The MCP detection efficiency for Ly- $\alpha$ can be estimated through $\varepsilon_{\mathrm{MCP}}=\mathrm{OAR} \cdot \varepsilon_{\mathrm{CsI}}$, where OAR stands for the openarea-ratio of the MCP itself and is 0.45 in our case. The quantum yield of the conversion from Ly- $\alpha$ to an electron in the CsI, $\varepsilon_{\text {CsI }}$, is in the range of $0.45-0.55[46,47]$. This leads to

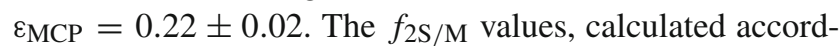
ing to Eq. 1, are summarized in Table 1 for $E_{\text {inc }}$ of 7.5 and $10 \mathrm{keV}$. Stronger scattering of the muon beam by the foil at $5 \mathrm{keV} E_{\text {inc }}$ prevented us from obtaining the reliable triplecoincidence signal needed to extract the $2 \mathrm{~S}$ fraction. Assuming, in accordance with hydrogen in a comparable velocity range (see Fig. 3.1 of [32]), that the $2 \mathrm{~S}$ fraction is nearly constant above $1 \mathrm{keV}$, we obtain a weighted average value of $f_{2 \mathrm{~S} / \mathrm{M}}=10 \pm 2 \%$. This value agrees with estimations in the literature which span $10-13 \%$ in this energy range $[18,26,32]$.

\section{Metastable beam intensity and Lamb shift outlook}

During the beam time, the proton current at the pion production target was $1.6 \mathrm{~mA}$ and the corresponding rate of 
Table 1 Summary of values extracted from different incident energies $E_{\text {inc }}$. MPE is the Most Probable Energy for M that traversed the foil and reached the Stop-MCP

\begin{tabular}{llllll}
\hline $\begin{array}{l}E_{\mathrm{inc}} \\
(\mathrm{keV})\end{array}$ & $\begin{array}{l}\text { MPE } \\
(\mathrm{keV})\end{array}$ & $\begin{array}{l}f_{\mathrm{M} / \mu^{+}} \\
(\%)\end{array}$ & $\begin{array}{l}f_{2 \mathrm{~S} / \mathrm{M}} \\
(\%)\end{array}$ & $\begin{array}{l}R_{\mu^{+}} \\
(\mathrm{kHz})\end{array}$ & $\begin{array}{l}R_{2 \mathrm{~S}} \\
(\mathrm{~Hz})\end{array}$ \\
\hline 5.0 & $2.7 \pm 0.1$ & $56.8 \pm 9.0$ & - & 1.45 & $83^{*} \pm 21$ \\
7.5 & $4.7 \pm 0.2$ & $43.2 \pm 2.4$ & $11 \pm 4$ & 2.07 & $100 \pm 30$ \\
10.0 & $7.0 \pm 0.3$ & $31.8 \pm 0.8$ & $10 \pm 3$ & 2.84 & $90 \pm 30$ \\
\hline
\end{tabular}

For $R_{2 \mathrm{~S}}$ at $5 \mathrm{keV}, f_{2 \mathrm{~S} / \mathrm{M}}=10 \pm 2 \%$ was assumed (see text)

moderated $\mu^{+}$emerging was $18 \mathrm{kHz}$. Using the LEM beamline simulation $[39,40]$, with the same conditions as in our experiment, we estimate the rate of $\mu^{+}$passing the foil, $R_{\mu^{+}}$, for each $E_{\text {inc }}$. The rate of metastable $\mathrm{M}$ is obtained by multiplying with the measured formation efficiencies, $R_{2 \mathrm{~S}}=R_{\mu^{+}} f_{\mathrm{M} / \mu^{+}} f_{2 \mathrm{~S} / \mathrm{M}}$. The results are given in Table 1 . As shown in Figure $5, f_{\mathrm{M} / \mu^{+}}$decreases by increasing the beam energy. Nevertheless, the angular distribution of the beam emerging from the foil at $10 \mathrm{keV}$ is narrower, thus, the transmission of the beamline increases, such that the final metastable rates are comparable. Therefore, we concentrate on this energy for considering the rates available for future experiments.

In Fig. 1, the main missing component for precision spectroscopy experiments of the Muonium Lamb shift and fine structure is a broadband microwave apparatus that we would place in the extension stage. By mixing the population with the $2 \mathrm{P}$ states, this could then resonantly quench the $2 \mathrm{~S}$ beam. In this 'opt-out' scheme, the Ly- $\alpha$ signal decreases near to the resonance.

Focusing on the Lamb shift transitions, we would obtain a clean symmetric line shape of the resonance by driving the 2S $F=0 \rightarrow 2 \mathrm{P}_{1 / 2} F=1$ transition around $580 \mathrm{MHz}$. This is isolated from the next transition by $0.6 \mathrm{GHz}$, which is favorable to that of hydrogen where the difference is only 0.2 GHz. Based on minor improvements to the setup presented here, the expected rate of detected $\mathrm{M}(2 \mathrm{~S})$ states will be $8 / \mathrm{s}$. This is four orders of magnitude larger than the rate of $5 / \mathrm{h}$ measured at TRIUMF [48], and 4/h at LAMPF [27]. A further increase in slow muon rate by roughly a factor 50 is expected once the muCool beamline comes online [49-51].

To prevent the $2 \mathrm{~S} F=1$ levels from contributing to the background we would introduce a hyperfine selection stage in front of the microwave cavity in the extension section, which deexcites most of the $2 \mathrm{~S} F=1$ population to the ground state, leaving a clean beam of roughly $22 / \mathrm{s} \mathrm{M}(2 \mathrm{~S})$ $F=0$, and an off-resonance coincidence signal of $2 / \mathrm{s}$. At this rate, with 120 hours of beamtime, the $100 \mathrm{MHz}$ natural linewidth could be resolved to $0.1 \mathrm{MHz}$.

\section{Conclusions}

We have demonstrated the creation of an intense directed beam of Muonium in the long-lived 2S state by transmitting low energy muons from the LEM beamline through a thin carbon foil. With an estimation of the $\mu^{+}$rate as well as the measurement of the neutral and $2 \mathrm{~S}$ fractions (see Table 1), we determined a conversion rate of $(3 \pm 1) \% \mathrm{M}(2 \mathrm{~S})$ per incoming $\mu^{+}$at $10 \mathrm{keV}$. This opens up the possibility to conduct precision measurements of laser and microwave transitions from the $\mathrm{M}(2 \mathrm{~S})$ state. For a measurement of the $n=2 \mathrm{Lamb}$ shift, arguably the most promising of these transitions, an uncertainty on the order of $100 \mathrm{kHz}$ is projected, which constitutes an improvement by two orders of magnitude over the best determination from the literature [18].

A determination of the Lamb shift in muonium with this accuracy will provide a stringent test of high-order recoil corrections in bound state QED [52,53], free of finite-size effects [1]. Moreover, it will be a sensitive probe for the existence of exotic dark-sector particles [54], new muonic forces [55], and hidden dimensions [56,57].

Acknowledgements The work presented here is supported by the European Research Council (Grant number 818053-Mu-MASS), the Yamada Science Foundation, and ETH Zürich (Grant number ETH-46 17-1). BO is supported by the Israel Academy of Sciences and Humanities. We would like to acknowledge the contributions of D. Nandal, A. Nanda and J. Zhang during various stages of this work. The muon measurements have been performed at the Swiss Muon Source $\mathrm{S} \mu \mathrm{S}$, Paul Scherrer Institute, CH-5232 Villigen, Switzerland.

Data Availability Statement This manuscript has no associated data or the data will not be deposited. [Authors' comment: Data is available from the authors upon request.]

Open Access This article is licensed under a Creative Commons Attribution 4.0 International License, which permits use, sharing, adaptation, distribution and reproduction in any medium or format, as long as you give appropriate credit to the original author(s) and the source, provide a link to the Creative Commons licence, and indicate if changes were made. The images or other third party material in this article are included in the article's Creative Commons licence, unless indicated otherwise in a credit line to the material. If material is not included in the article's Creative Commons licence and your intended use is not permitted by statutory regulation or exceeds the permitted use, you will need to obtain permission directly from the copyright holder. To view a copy of this licence, visit http://creativecomm ons.org/licenses/by/4.0/.

Funded by $\mathrm{SCOAP}^{3}$.

\section{References}

1. S.G. Karshenboim, Phys. Rep. 422(1), 1 (2005). https://doi.org/10. 1016/j.physrep.2005.08.008

2. T. Blum, A. Denig, I. Logashenko, E. de Rafael, B.L. Roberts, T. Teubner, G. Venanzoni. arXiv preprint (2013) 
3. R. Pohl, R. Gilman, G.A. Miller, K. Pachucki, Annu. Rev. Nucl. Part. Sci. 63(1), 175 (2013). https://doi.org/10.1146/ annurev-nucl-102212-170627

4. P.J. Mohr, D.B. Newell, B.N. Taylor, Rev. Mod. Phys. 88, 035009 (2016). https://doi.org/10.1103/RevModPhys.88.035009

5. H. Fleurbaey, S. Galtier, S. Thomas, M. Bonnaud, L. Julien, F. Biraben, F. Nez, M. Abgrall, J. Guéna, Phys. Rev. Lett. 120, 183001 (2018). https://doi.org/10.1103/PhysRevLett.120.183001

6. J. Arrington, I. Sick, J. Phys. Chem. Ref. Data 44(3), 031204 (2015). https://doi.org/10.1063/1.4921430

7. A. Beyer, L. Maisenbacher, A. Matveev, R. Pohl, K. Khabarova, A. Grinin, T. Lamour, D.C. Yost, T.W. Hänsch, N. Kolachevsky, T. Udem, Science 358(6359), 79 (2017). https://doi.org/10.1126/ science.aah6677

8. N. Bezginov, T. Valdez, M. Horbatsch, A. Marsman, A.C. Vutha, E.A. Hessels, Science 365(6457), 1007 (2019). https://doi.org/10. 1126/science.aau 7807

9. W. Xiong, A. Gasparian, H. Gao, D. Dutta, M. Khandaker, N. Liyanage, E. Pasyuk, C. Peng, X. Bai, L. Ye et al., Nature 575(7781), 147 (2019). https://doi.org/10.1038/ s41586-019-1721-2

10. K. Jungmann, Hyperfine Interact. 127, 189 (2000). https://doi.org/ 10.1023/A:1012639500419

11. K. Jungmann, J. Phys. Soc. Jpn. 85(9), 091004 (2016). https://doi. org/10.7566/JPSJ.85.091004

12. S. Chu, A.P. Mills, A.G. Yodh, K. Nagamine, Y. Miyake, T. Kuga, Phys. Rev. Lett. 60, 101 (1988). https://doi.org/10.1103/ PhysRevLett.60.101

13. V. Meyer et al., Phys. Rev. Lett. 84, 1136 (2000). https://doi.org/ 10.1103/PhysRevLett.84.1136

14. P. Crivelli, Hyperfine Interact. 239(1), 49 (2018). https://doi.org/ 10.1007/s10751-018-1525-z

15. W. Liu et al., Phys. Rev. Lett. 82, 711 (1999). https://doi.org/10. 1103/PhysRevLett.82.711

16. Y. Ueno et al., Hyperfine Interact. 238(1), 14 (2017). https://doi. org/10.1007/s10751-016-1381-7

17. P. Strasser, et al., in EPJ Web of Conferences, vol. 198 (2019), vol. 198, p. 3. https://doi.org/10.1051/epjconf/201919800003

18. C.J. Oram, J.M. Bailey, P.W. Schmor, C.A. Fry, R.F. Kiefl, J.B. Warren, G.M. Marshall, A. Olin, Phys. Rev. Lett. 52, 910 (1984). https://doi.org/10.1103/PhysRevLett.52.910

19. K. Woodle et al., Phys. Rev. A 41, 93 (1990). https://doi.org/10. 1103/PhysRevA.41.93

20. S.H. Kettell, Measurement of the $2 \mathrm{~s} 1 / 2$ - 2p3/2 fine structure interval in muonium. Ph.D. Thesis, Yale University (1990). https://doi. org/10.2172/6849854

21. C.W. Fabjan, F.M. Pipkin, M. Silverman, Phys. Rev. Lett. 26, 347 (1971). https://doi.org/10.1103/PhysRevLett.26.347

22. C.W. Fabjan, F.M. Pipkin, Phys. Rev. A 6, 556 (1972). https://doi. org/10.1103/PhysRevA.6.556

23. B.O. Clark, D.A. Van Baak, S.R. Lundeen, F.M. Pipkin, Phys. Rev. A 19, 802 (1979). https://doi.org/10.1103/PhysRevA.19.802

24. E. Arnold, T. Kühl, E.W. Otten, L.V. Reisky, Phys. Lett. A 90(8), 399 (1982). https://doi.org/10.1016/0375-9601(82)90794-0

25. H.J. Andrä, Phys. Scr. 9(5), 257 (1974). https://doi.org/10.1088/ 0031-8949/9/5/002

26. C. Oram, C.A. Fry, J.B. Warren, R.F. Kiefl, J.H. Brewer, J. Phys. B Atom. Mol. Phys. 14(23), L789 (1981). https://doi.org/10.1088/ 0022-3700/14/23/007

27. A. Badertscher et al., Formation of Muonium in the $2 \mathrm{~S}$ state and observation of the lamb shift transition, pp. 233-236 (1984). https:// doi.org/10.1142/9789814343107_0031

28. H. Massey, in Atoms II/Atome II Springer, pp. 232-306 (1956). https://doi.org/10.1007/978-3-642-85687-7_3

29. P.R. Bolton et al., Phys. Rev. Lett. 47, 1441 (1981). https://doi.org/ 10.1103/PhysRevLett.47.1441
30. T. Prokscha, E. Morenzoni, M. Meyberg, T. Wutzke, B.E. Matthias, A. Fachat, K. Jungmann, G. zu Putlitz, Phys. Rev. A 58, 3739 (1998). https://doi.org/10.1103/PhysRevA.58.3739

31. H.E. Ahn, Part 1, angular distribution measurement of beam-foil muonium, part 2, muon injection simulation for a new muon $\mathrm{g}-2$ experiment. Ph.D. Thesis, Yale University (1992)

32. C.A. Fry, Measurement of the lamb shift in muonium. Ph.D. Thesis, University of British Columbia (1985). https://doi.org/10.14288/ 1.0085236

33. T. Prokscha, E. Morenzoni, K. Deiters, F. Foroughi, D. George, R. Kobler, V. Vranković, Hyperfine Interact. 159, 385 (2004). https:// doi.org/10.1007/s10751-005-9129-9

34. T. Prokscha, E. Morenzoni, K. Deiters, F. Foroughi, D. George, R. Kobler, A. Suter, V. Vrankovic, Nucl. Instrum. Methods A $\mathbf{5 9 5}$ 317 (2008). https://doi.org/10.1016/j.nima.2008.07.081

35. D.R. Harshman, A.P. Mills, J.L. Beveridge, K.R. Kendall, G.D. Morris, M. Senba, J.B. Warren, A.S. Rupaal, J.H. Turner, Phys. Rev. B 36, 8850 (1987). https://doi.org/10.1103/PhysRevB.36.8850

36. T. Prokscha, E. Morenzoni, C. David, A. Hofer, H. Glückler, L. Scandella, Appl. Surf. Sci. 172(3), 235 (2001). https://doi.org/10. 1016/S0169-4332(00)00857-6

37. G. Gabrielse, Phys. Rev. A 23, 775 (1981). https://doi.org/10.1103/ PhysRevA.23.775

38. M. Gonin, R. Kallenbach, P. Bochsler, Rev. Sci. Instrum. 65(3), 648 (1994). https://doi.org/10.1063/1.1145132

39. K. Sedlak, R. Scheuermann, T. Shiroka, A. Stoykov, A. Raselli, A. Amato, Physics Procedia 30, 61 (2012). https://doi.org/10.1016/j. phpro.2012.04.040. 12th International Conference on Muon Spin Rotation, Relaxation and Resonance ( $\mu$ SR2011)

40. K. Khaw, A. Antognini, P. Crivelli, K. Kirch, E. Morenzoni, Z. Salman, A. Suter, T. Prokscha, J. Instrum. 10(10), P10025 (2015). https://doi.org/10.1088/1748-0221/10/10/p10025

41. F. Allegrini, R.W. Ebert, H.O. Funsten, J. Geophys. Res. Sp. Phys. 121(5), 3931 (2016). https://doi.org/10.1002/2016JA022570

42. C. Latimer, R. McMahon, D. Murtagh, Phys. Lett. A 87(5), 232 (1982). https://doi.org/10.1016/0375-9601(82)90010-X

43. F. Allegrini, R.W. Ebert, G. Nicolaou, G. Grubbs, Nucl. Instrum. Methods Phys. Res. Sect. B Beam Interact. Mater. Atoms 359, 115 (2015). https://doi.org/10.1016/j.nimb.2015.07.098

44. D.A. Dahl, Int. J. Mass Spectrom. 200(1), 3 (2000). https://doi. org/10.1016/S1387-3806(00)00305-5. Volume 200: The state of the field as we move into a new millenium

45. A. van Wijngaarden, G.W.F. Drake, Phys. Rev. A 17, 1366 (1978). https://doi.org/10.1103/PhysRevA.17.1366

46. A. Tremsin, O. Siegmund, Proc. SPIE (1999). https://doi.org/10. $1117 / 12.366524$

47. A. Tremsin, O. Siegmund, Proc SPIE 5920, (2005). https://doi.org/ $10.1117 / 12.621761$

48. C. Fry, J. Warren, R. Kiefl, C. Oram, G. Ludgate, P. Schmor, A. Olin, G. Marshall, B. Erickson, G. Morris, Hyperfine Interact. 18(1-4), 691 (1984). https://doi.org/10.1007/BF02064887

49. I. Belosevic, A. Antognini, Y. Bao, A. Eggenberger, M. Hildebrandt, R. Iwai, D.M. Kaplan, K.S. Khaw, K. Kirch, A. Knecht et al., Hyperfine Interact. 240(1), 41 (2019)

50. I. Belosevic, A. Antognini, Y. Bao, A. Eggenberger, M. Hildebrandt, R. Iwai, D. Kaplan, K.S. Khaw, K. Kirch, A. Knecht et al., Eur. Phys. J. C 79(5), 430 (2019)

51. A. Antognini, N. Ayres, I. Belosevic, V. Bondar, A. Eggenberger, M. Hildebrandt, R. Iwai, D. Kaplan, K. Khaw, K. Kirch, et al., arXiv preprint arXiv:2003.11986 (2020)

52. K.P. Jungmann. Muonium (1998). arXiv:physics/9809020 [physics.atom-ph]

53. V.A. Yerokhin, K. Pachucki, V. Patkóš, Ann. Phys. 531(5), 1800324 (2019). https://doi.org/10.1002/andp.201800324

54. C. Frugiuele, J. Pérez-Ríos, C. Peset, Phys. Rev. D 100, 015010 (2019). https://doi.org/10.1103/PhysRevD.100.015010 
55. B. Batell, D. McKeen, M. Pospelov, Phys. Rev. Lett. 107, 011803 (2011). https://doi.org/10.1103/PhysRevLett.107.011803

56. L. Zhi-Gang, N. Wei-Tou, A.P. Patón, Chin. Phys. B 17(1), 70 (2008). https://doi.org/10.1088/1674-1056/17/1/013
57. F. Dahia, A.S. Lemos, Phys. Rev. D 94, 084033 (2016). https://doi. org/10.1103/PhysRevD.94.084033 